\title{
Conhecendo a flora herbáceo-subarbustiva do Parque Estadual do Mirador, Maranhão/Brasil
}

\author{
Mauricio Santos da Silva ${ }^{1, *}$ (D), Thamires Oliveira Reis' (D), Laiza Oliveira Silva' (D), \\ Abilene Espindola Correia' (D), Arthur Filipe Mendes Couto' (1), \\ Raysa Valéria Carvalho Saraiva ${ }^{2}$ (i) \& Francisca Helena Muniz'
}

\author{
'Universidade Estadual do Maranhão, Departamento de Biologia, Av. Lourenço Vieira da Silva, n¹000, Jardim São Cristóvão, \\ 65055-310, São Luís, Maranhão, Brasil. \\ *Autor para correspondência: mausantosdasilva@gmail.com \\ 2Universidade Federal do Maranhão, Centro de Ciências Humanas, Naturais, Saúde e Tecnologia, Estrada de Pacas,s/n, Enseada, \\ 65200-000, Pinheiro, Maranhão, Brasil.
}

Recebido em 16.IX.2019

Aceito em 05.X.2021

DOI 10.21826/2446-82312022v77e2022002

\begin{abstract}
RESUMO- Realizamos um estudo florístico no Cerrado stricto sensu do Parque Estadual do Mirador na região centro-sul do estado do Maranhão, com objetivo de conhecer as espécies que compõem a vegetação herbáceo-subarbustivo e comparar a riqueza florística com outras áreas do Cerrado brasileiro. Para tanto, coletamos materiais botânicos em parcelas estabelecidas em campo e fora delas para uma melhor representação da flora. Para a análise de similaridade entre diferentes áreas de Cerrado foram selecionados trabalhos publicados referentes a vegetação herbáceo-subarbustiva de áreas de Unidade de Conservação, em seguida montada uma matriz binária para efetuar a análise estatística. As famílias que apresentaram maior número de espécies foram Fabaceae (18), Cyperaceae (17), Malvaceae (7) e Arecaceae (6). Das espécies catalogadas no PEM, 47\% eram ervas, 41\% subarbusto e $14 \%$ trepadeira. As áreas de Cerrado comparadas com a vegetação herbáceo-subarbustivo do PEM apresentaram baixa similaridade, caracterizando distinção florística entre as áreas.
\end{abstract}

Palavras-Chave: Cerrado, riqueza, similaridade

ABSTRACT- Knowing the herb-subshrub flora of Mirador State Park, Maranhão / Brazil. We carried out a floristic study of the Cerrado stricto sensu flora of the Mirador State Park in the center-south region of the State of Maranhão, aiming to know the species that make up its herbaceoussubshrub vegetation and to compare its floristic richness with these of other areas of Brazilian Cerrado. We collected botanical materials in plots established inside and outside of the area, for a better representation of the flora. To assess the similarity between different areas of Cerrado, we selected published papers referring to herbaceous-subshrub vegetation of Conservation Unit areas and then assembled a binary matrix to perform the statistical analysis. The families with the highest number of species were Fabaceae (18), Cyperaceae (17), Malvaceae (7) and Arecaceae (6). Of the species catalogued in PEM, 47\% were herbs, $41 \%$ subshrubs and 14\% climbers. The other Cerrado areas showed low similarity with PEM, characterizing a floristic distinction between the areas.

Keywords: Cerrado, richness, similarity

\section{INTRODUÇÃO}

O Cerrado no Estado do Maranhão, nordeste do Brasil, corresponde a aproximadamente $40 \%$ do território maranhense, englobando várias formas de vegetação com diferentes tipos estruturais, relacionados principalmente a um gradiente de biomassa variando desde o campo sujo, campo cerrado, cerrado (stricto sensu), até cerradão, para os quais a interação de fatores climáticos, topográficos e edáficos, além da presença de fogo, são considerados determinantes em sua ocorrência (Muniz 2006).

Dentre as áreas de Cerrado protegidas no Maranhão, o Parque Estadual do Mirador (PEM) é a mais extensa com 766.781,00 ha, e compreende os municípios de Mirador, Formosa da Serra Negra e Fernando Falcão (Maranhão
2009). A área do PEM tem o Cerrado sensu lato como vegetação predominante (Conceição \& Castro 2009), está situada numa zona de transição Cerrado-Amazônia, sendo área relevante para conservação da biodiversidade do bioma (MMA 2007). O Cerrado é caracterizado por apresentar maior dimensão de espécies herbáceas comparadas às arbóreas (Batalha \& Mantovani 2001, Mendonça et al. 2001, 2008, Rios 2016). Entretanto, existem mais trabalhos publicados sobre estudos florísticos, fitossociológicos e fenológicos acerca da vegetação arbórea.

As características do estrato herbáceo-subarbustivo é um fator considerado para classificação fisionômica do Cerrado, onde são levadas em consideração as estruturas, a mudança no aspecto vegetativo durante o ano, a forma de crescimento, a consistência e o tamanho das folhas 
(Munhoz \& Felfili 2006). Vários autores (Tannus \& Assis 2004; Munhoz \& Felfili 2005, 2006, 2007) afirmam que a flora herbáceo-subarbustiva apresenta uma grande riqueza de espécies que ainda necessita de pesquisas sobre a composição florística, fitossociológica e comparação de similaridade entre áreas de Cerrado.

Diante disso, os levantamentos florísticos servem de subsídio para o manejo, a preservação e a conservação de ecossistemas, representando todas as espécies presentes na área sem distinção ecológica (Muniz 2006), e são um recurso útil à pesquisa botânica e ecológica, onde permite qualificar a fitodiversidade do local e desenvolver metaanálises para estudos biogeográficos.

Os padrões qualitativos da vegetação são conhecidos ou determinados por meio de levantamentos florísticos, caracterizando a riqueza, diversidade e similaridade dentro das áreas em questão, com capacidade de comparar as variações florísticas da vegetação e identificar as possíveis diferenças que podem existir entre áreas estudadas (Silva, 2016).

Este trabalho teve como objetivo analisar a composição florística do estrato herbáceo-subarbustivo do Parque Estadual do Mirador, por meio da caracterização da riqueza florística e comparando a similaridade com outras áreas de cerrado.

\section{MATERIAL E MÉTODO}

\section{Área de estudo}

Os levantamentos florísticos foram realizados no Parque Estadual do Mirador (PEM) (Fig. 1), próximo ao Posto do Mel, sob as seguintes coordenadas: $6^{\circ} 43^{\prime} 20,70^{\prime \prime} \mathrm{S}$ e $44^{\circ} 59^{\prime} 35,51^{\prime \prime} \mathrm{W}$. As coletas foram realizadas nos meses de agosto (2016), fevereiro (2017), agosto (2017) e novembro (2017), com esforço amostral de 3 a 4 dias por coleta. Foi obtida a licença de coleta $n^{\circ}$ 194229/2014 da Secretaria de Estado do Meio Ambiente e Recursos Renováveis.

O Parque Estadual do Mirador (PEM) está localizado na região centro-meriodional do estado do Maranhão $\left(06^{\circ} 10^{\prime}-06^{\circ} 42\right.$ 'S e $44^{\circ} 43^{\prime}-45^{\circ} 54$ 'W,425 asl, WGS84 datum), Brasil, entre as cabeceiras do rio Itapecuru e rios Alpercartas, e engloba os municípios de Mirador, Formosa da Serra Negra e Fernando Falcão. Criado em 1980 por Decreto Estadual n ${ }^{\circ}$. 641, de 20 de junho, o PEM tem o Cerrado sensu lato (Fig. 2) como vegetação predominante (Conceição \& Castro 2009) em latossolos vermelhoamarelos associados a areias de quartzo e solos líticos, pedregosos e rochosos. Com uma área inicial de 700.000 ha, o PEM foi ampliado pela Lei ${ }^{\circ}$. 8.958, de 08 de maio de 2009 , tendo agora uma área total de 766.781,00 ha
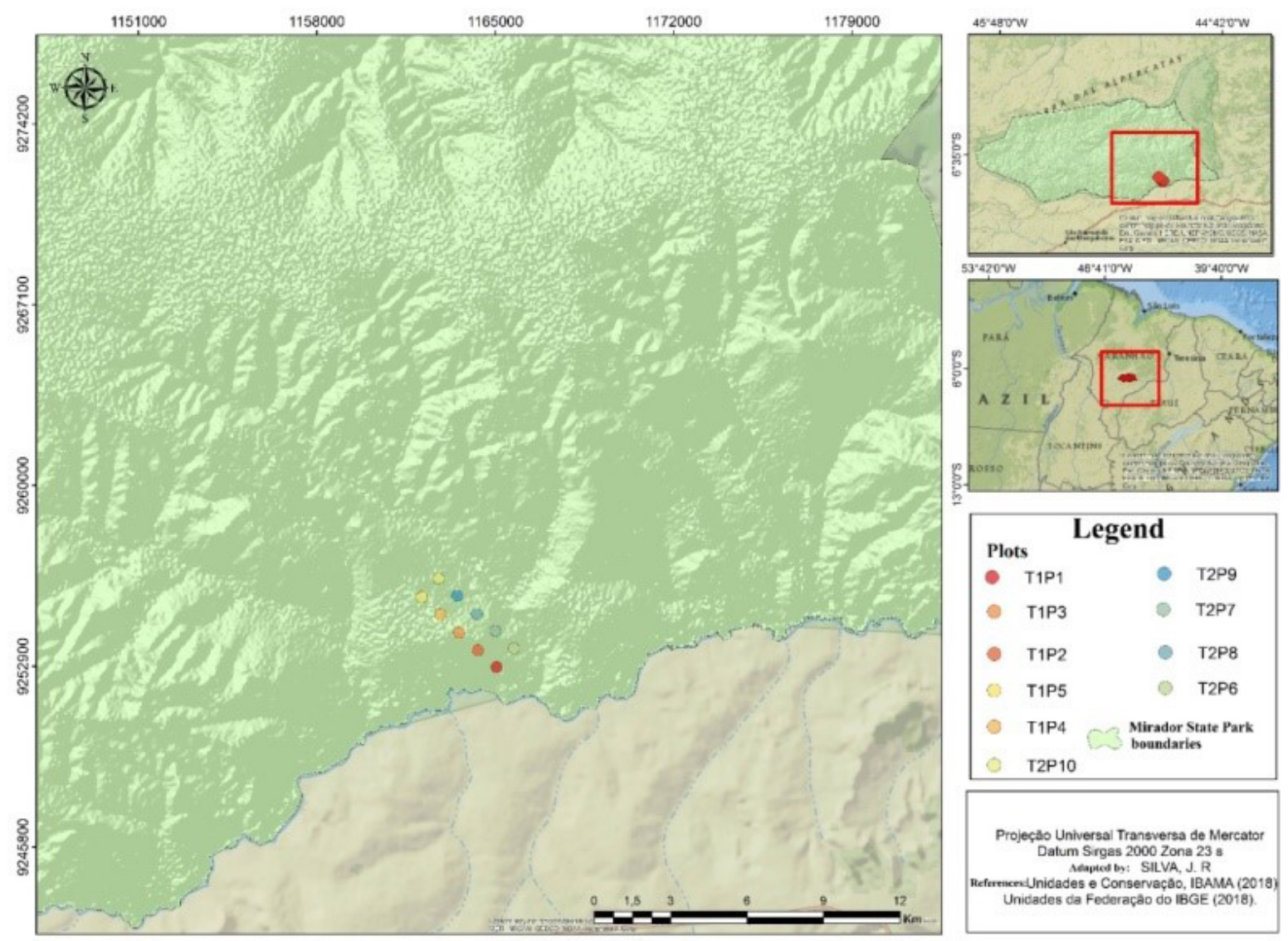

Figura 1. Localização do Parque Estadual do Mirador e a área de coleta. 
(Maranhão 2009). O clima é do tipo Aw (tropical subhúmido seco) com precipitação anual 1.200-1.400 mm e temperaturas médias variando de $19,5^{\circ}$ a $33^{\circ} \mathrm{C}$ (Alcântara 2004, Andrade et al. 2017). O período chuvoso corresponde aos meses dezembro, janeiro, fevereiro, março, abril, maio; a estação seca aos meses, Junho, Julho, Agosto, Setembro, Outubro e Novembro.

\section{Amostragem da vegetação}

A coleta de ramos férteis foi realizada nos limites estabelecidos das parcelas já alocadas no Parque Estadual do Mirador para vegetação arbórea do Projeto PPBio Rede ComCerrado, na fitofisionomia stricto sensu, cada parcela apresenta 25 segmentos de 10 metros, totalizando 250 metros de comprimento e largura de 40 metros, foram alocadas 10 parcelas em duas trilhas de $5 \mathrm{~km}$ (5 parcelas em cada trilha) próximo ao Posto do Mel e também ao longo das trilhas das parcelas foram feitas coletas. As técnicas de coleta e herborização utilizaram os métodos usuais desenvolvidos no Herbário Rosa Mochel-UEMA. O material coletado foi encaminhado para estufa, para desidratação.

Os materiais botânicos férteis (com flores ou frutos) coletados em campo foram incluídos no acervo e registrados no Herbário Rosa Mochel, e para essa listagem foram incrementadas espécies herbáceo-subarbustivas presentes no herbário, considerando apenas os espécimes já coletados no Parque Estadual do Mirador (PEM) na fitofisionomia Cerrado stricto sensu.

\section{Identificação dos vegetais}

As espécies coletadas no PEM foram identificadas até família por meio de chaves disponíveis no Angiosperm Phylogeny Group IV (Byng et al. 2016). A identificação a nível de espécie foi realizada por comparação com exemplares disponíveis no Herbário Rosa Mochel (SLUI) da Universidade Estadual do Maranhão Campus Paulo VI (UEMA), sites botânicos (Flora do Brasil 2020, Tropicos e SpeciesLink), consultas a bibliografias especializadas para cada família botânica (monografias, dissertações, teses e artigos) e consultas a especialistas. Quanto ao hábito do indivíduo herbáceo-subarbustivo foi determinado por meio de consultas no site Reflora (Reflora.jbrj.gov.br) e de acordo com a classificação de Whittaker (1975). A confirmação dos nomes científicos foi realizada através de consultas no site Tropicos (Tropicos.org/).

\section{Análise de Similaridade}

Para a análise de similaridade de riqueza entre áreas de Cerrado, foram selecionados trabalhos recentes publicados referentes a flora herbácea-subarbustiva realizados no Cerrado (Tabela 1), em seguida montada uma matriz binária de ausência e presença. Foi utilizado o método de ordenação não métrico (NMDS) por meio do Programa Past versão 3.18 beta, que dispõe as parcelas em um espaço multidimensional baseado na incidência de espécies, com a utilização das medidas de similaridade de Jaccard para análise de incidência (Mccune \& Grace, 2002).

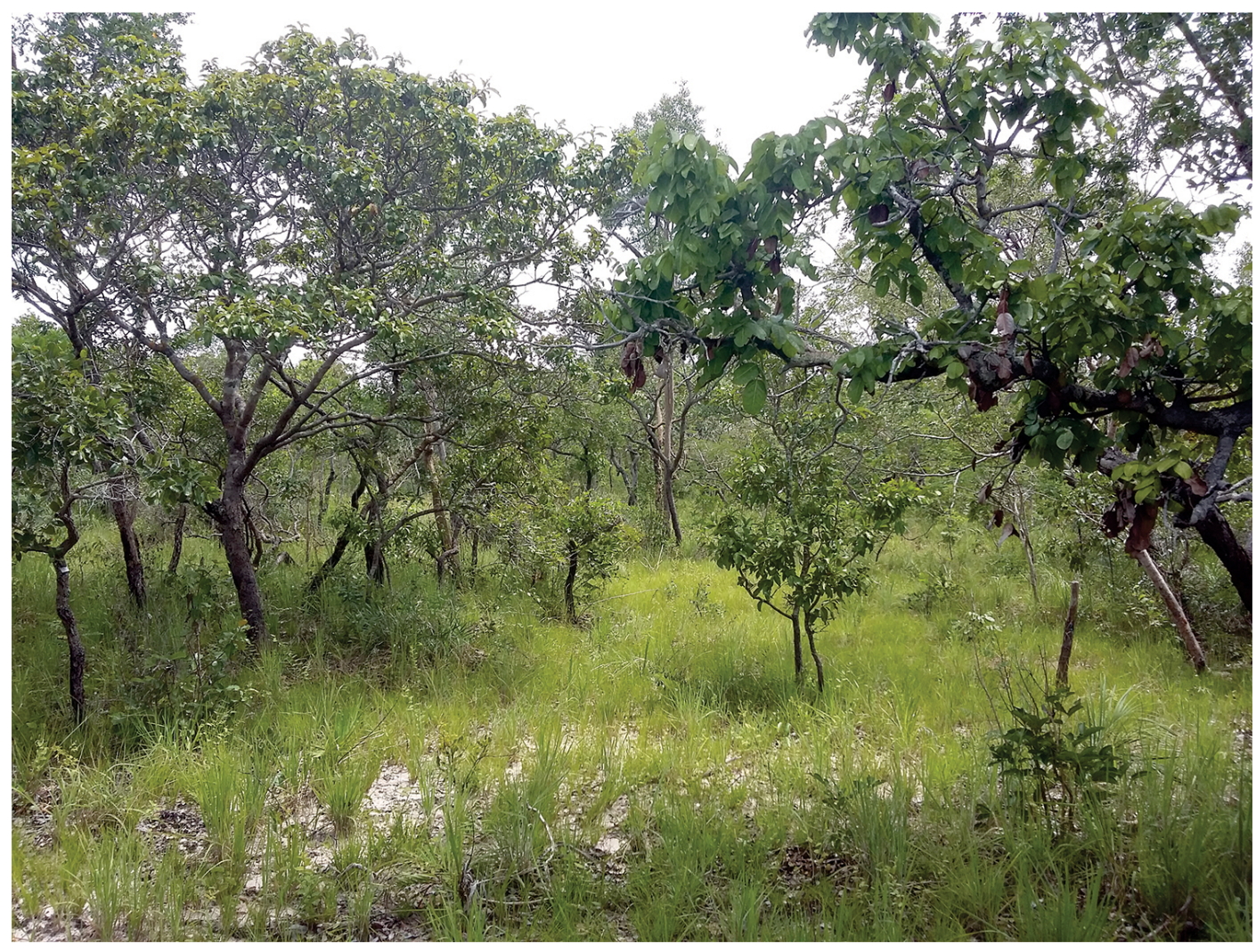

Figura 2. Vegetação característica do Parque Estadual de Mirador (PEM) 
Tabela 1. Trabalhos sobre a vegetação herbáceo-subarbustiva do Cerrado para análise de similaridade.

\begin{tabular}{lcc}
\hline Título do Artigo & Fitofisionomia & Referência \\
\hline $\begin{array}{l}\text { Composição, Estrutura e Diversidade da Vegetação Herbáceo-Arbustiva em } \\
\text { Veredas no Jalapão, Tocantins }\end{array}$ & Vereda & Silva (2016) \\
$\begin{array}{l}\text { Fatores modeladores da composição e da diversidade de espécies herbáceo- } \\
\text { Arbustivas em Veredas no Brasil }\end{array}$ & Vereda & Bijos (2017) \\
$\begin{array}{l}\text { Florística e estrutura da vegetação em Cerrado sentido restrito no Parque } \\
\text { Estadual de Terra Ronca, Goiás: método RAPELD }\end{array}$ & Stricto sensu & Teixeira (2015) \\
$\begin{array}{l}\text { Levantamento Florístico dos Arredores do Parque das Capivaras, Três } \\
\text { Lagoas-MS }\end{array}$ & Mata de Galaria & Andrella e Neto (2017) \\
$\begin{array}{l}\text { Comunidades Herbáceo-Arbustivas e suas relações com o solo e altitude, em } \\
\text { área secas e úmidas, no Parque Nacional das Sempre Vivas, MG } \\
\text { Checklist da flora herbáceo-arbustiva da Reserva Biológica do Guará, } \\
\begin{array}{l}\text { Distrito Federal: levantamento da flora como parte integrante de estudos } \\
\text { para subsidiar o manejo e gestão de áreas protegidas. }\end{array}\end{array}$ & Vereda e Campo Úmido \\
\hline
\end{tabular}

\section{RESULTADOS E DISCUSSÃO}

No Parque Estadual do Mirador (PEM), no Cerrado stricto sensu foram catalogadas 118 espécies inseridas em 89 gêneros e 40 famílias para o componente herbáceosubarbustivo (Tabela 2, Figs. 5, 6, 7 e 8). Embora a composição florística entre diferentes fitofisionomias do Cerrado varie, o número de espécies é parecido. A lista de espécies deste trabalho mostrou um número semelhante quando comparado aos registrados por Munhoz \& Felfili (2007) em campo limpo úmido no Distrito Federal, que amostraram 197 espécies. Já Rezende (2007), no Tocantins, amostrou um total de 136 espécies.

As famílias com maior número de espécies foram Fabaceae (18), Cyperaceae (17), Malvaceae (7), Arecaceae (6), Passifloraceae (5), Poaceae (5), Rubiaceae (5), Convolvulaceae (4) e Euphorbiaceae (4) (Fig. 3). Teixeira et al. (2016) destacam que as famílias Fabaceae, Rubiaceae e Cyperaceae apresentaram uma maior riqueza de espécies no Cerrado sensu stricto no Parque Estadual de Terra Ronca-Goiás; Bijos et al. (2017a) e Silva et al. (2017) ressaltam a família Cyperaceae como uma das que possui maior número de espécies para o componente herbáceosubarbustivo em trabalho feito na fitofisionomia vereda no Brasil Central e na Estação Ecológica Serra Geral do Tocantins, Chacon et al. (2014) afirma que as famílias Fabaceae, Rubiaceae, Cyperaceae e Euphorbiaceae com maior número de espécies em estudo na Reserva Biológica do Guará, Distrito Federal, em diferentes fitofisionomias do cerrado. Amaral et al. (2017), em levantamentos florísticos no Cerrado, destacaram a família Fabaceae como uma das mais ricas da região.

Segundo Carvalho et al. (2008), Fabaceae é uma das famílias botânicas mais diversificadas das províncias fitogeográficas brasileiras e apresenta um registro frequente entre as famílias mais importantes em áreas de Cerrado sensu stricto. De acordo com Embrapa (2012) essa riqueza está associada a capacidade que algumas espécies possuem em se associar a bactérias diazotróficas, fixadoras de nitrogênio atmosférico, e a família Fabaceae apresenta maior expressividade neste atributo. Segundo Teixeira (2015) essa especialidade proporciona a ela estabelecimento em áreas com diferentes condições ambientais. Cardoso \& Queiroz (2010) afirmam, também, que esta maior riqueza está relacionada a adaptações morfológicas a locais que apresentam estresse ambiental, como baixa disponibilidade de água e altas temperaturas. Essa preferência por ambientes secos e sazonais está relacionada ao fato de plantas da família Fabaceae possuir adaptações morfológicas capazes de suportarem o estresse hídrico, entre outras famílias do Cerrado.

Os gêneros mais representativos em número de espécies foram Chamaecrista (7), Evolvulus (3) e Euphorbia (3). Fernandes \& Garcia (2008) e Andrella \& José Neto (2017) destacam o gênero Chamaecrista entre os que apresentam maior riqueza de espécies em estudos no Cerrado.

Das espécies identificadas no PEM, 59 eram ervas, 41 subarbustos e 19 trepadeiras. Rios (2016) destaca a forma de vida subarbustiva com maior representação em relação a erva em estudos no Cerrado típico no Distrito Federal, enquanto o hábito trepadeira apresentou baixa porcentagem comparada às outras formas de crescimento. Amaral et al. (2013) apontam este mesmo resultado em pesquisa no cerrado do Distrito Federal, mas o hábito com maior número de espécies descrito foi o do tipo erva.

Dez espécies catalogadas (Aspilia leucoglossa Malme, Ayenia angustifolia A. ST.-Hil. \& Naudin, Banisteriopsis stellaris (Griseb.) B. Gates, Croton mucronifolius Müll. Arg., Evolvulus anagalloides Meisn., Evolvulus pusillus Choisy, Gomphrena agrestis Mart., Ichthyothere latifolia Baker, Periandra coccinea (Schrader.) Benth., Symphyllophyton campos-portoi Gilg-Bem., Habranthus sylvaticus Herb.) são endêmicas do território brasileiro, correspondendo a $15,87 \%$ das espécies coletadas no Parque Estadual do Mirador, destas, duas (A. leucoglossa, I. latifolia) são endêmicas do Cerrado, de acordo com a Flora do Brasil 2020 (2019). 
Tabela 2. Espécies da flora vascular, em ordem de família, em área de Cerrado sensu stricto do Parque Estadual do Mirador (PEM), Maranhão Brasil e das espécies depositadas no acervo do herbário SLUI que não foram coletadas durante a realização deste trabalho.

\begin{tabular}{|c|c|c|c|c|c|}
\hline Família/Espécie & Nome Comum & Floração & Frutificação & Hábito & SLUI \\
\hline \multicolumn{6}{|l|}{ Acanthaceae } \\
\hline Ruellia gemniflora Kunth & - & - & - & Sub & 366 \\
\hline \multicolumn{6}{|l|}{ Amaranthaceae } \\
\hline Gomphrena agrestis Mart. & - & Ago. e Fev. & - & Sub & 4742 \\
\hline \multicolumn{6}{|l|}{ Amaryllidaceae } \\
\hline Habranthus sylvaticus Herb. & Flor-do-berrante & Nov. & - & Erva & 5109 \\
\hline Rhodolphiala bifida (Herb.) Traub & - & - & - & Erva & 4447 \\
\hline \multicolumn{6}{|l|}{ Apocynaceae } \\
\hline Galactophora sp. & - & - & - & Sub & 364 \\
\hline \multicolumn{6}{|l|}{ Arecaceae } \\
\hline Astrocaryum campestre Mart. & tucum-rasteiro & Ago. & Ago. e Nov. & Erva & 5012 \\
\hline Attalea spectabilis Mart. & - & - & - & Erva & 546 \\
\hline Geonoma brevispatha Barb. Rodr. & - & - & - & Erva & - \\
\hline Orbignya eichleri Drude & - & - & - & Erva & 545 \\
\hline Spathiphyllum sp. & - & - & - & Erva & 370 \\
\hline Syagrus comosa (Mart.) Mart. & pati & Ago. & - & Erva & 5013 \\
\hline \multicolumn{6}{|l|}{ Asteraceae } \\
\hline Aspilia leucoglossa Malme & - & Fev. & - & Sub & 4785 \\
\hline Ichthyothere latifolia Baker & - & Fev. & - & Sub & 4786 \\
\hline Vernonia $\mathrm{sp}$. & - & Ago. & - & Trep & 4788 \\
\hline \multicolumn{6}{|l|}{ Aristolochiaceae } \\
\hline Aristolochia didyma S. Moore & - & - & - & Trep & 214 \\
\hline \multicolumn{6}{|l|}{ Bignoniaceae } \\
\hline Fridericia platyphylla (Cham.) L.G. Lohmann & - & Fev. & - & Sub & 4789 \\
\hline \multicolumn{6}{|l|}{ Bromeliaceae } \\
\hline Ananas ananassoides (Baker) L. B. Sm. & - & - & - & Erva & 379 \\
\hline \multicolumn{6}{|l|}{ Caryophyllaceae } \\
\hline Polycarpaea corymbosa (L.) Lam. & - & Ago. e Fev. & - & Erva & 4787 \\
\hline \multicolumn{6}{|l|}{ Commelinaceae } \\
\hline Commelina erecta L. & - & Fev. & - & Erva & 4790 \\
\hline \multicolumn{6}{|l|}{ Convolvulaceae } \\
\hline Evolvulus anagalloides Meisn. & - & Fev. & - & Erva & 4792 \\
\hline Evolvulus frankenioides Moric. & - & Fev. & - & Erva & 4793 \\
\hline Evolvulus pusillus Choisy & - & Fev. & - & Trep & 4794 \\
\hline Bonamia agrostopolis Vell. & - & Fev. & - & Trep & 4791 \\
\hline \multicolumn{6}{|l|}{ Cyperaceae } \\
\hline Becquerelia cymosa Brongon & - & - & - & Erva & - \\
\hline Bulbostylis capillaris (L.) Kunth. Ex C. B. Clarke & barba-de-bode & Ago. e Fev. & - & Erva & 4739 \\
\hline Bulbostylis junciformis (Kunth.) & barba-de-bode & Ago. e Fev. & - & Erva & 4740 \\
\hline Bulbostylis spadicea (Kunth) Kük. & - & - & - & Erva & - \\
\hline Cyperus haspan L. & - & - & - & Erva & 396 \\
\hline Cyperus sp. & tiririca & Ago. e Fev. & - & Erva & 4741 \\
\hline Eleocharis interstincta (Vahl) Roem. \& Schult. & - & - & - & Erva & 385 \\
\hline Eleocharis minima Kunth & - & - & - & Erva & 4597 \\
\hline Fuirena incomplete Nees & - & - & - & Erva & 384 \\
\hline Fuirena umbellate Rottb & - & - & - & Erva & 398 \\
\hline Kyllinga odorata Vahl & - & Fev. & - & Erva & 4795 \\
\hline
\end{tabular}


Tabela 2. Cont.

\begin{tabular}{|c|c|c|c|c|c|}
\hline Família/Espécie & Nome Comum & Floração & Frutificação & Hábito & SLUI \\
\hline Lagenocarpus guianensis Nees & - & - & - & Erva & 386 \\
\hline Rhynchospora cephalotes (L.) Vahl & capim-do-brejo & Nov. & - & Erva & 5112 \\
\hline Rhynchospora exaltata Kunth & - & - & - & Erva & 395 \\
\hline Rhynchospora sp. & tiririca-do-brejo & Nov. & - & Erva & 5113 \\
\hline Scleria argentea Steud & - & - & - & Erva & 393 \\
\hline Scleria nitens Berg & - & - & - & Erva & 392 \\
\hline \multicolumn{6}{|l|}{ Dilleniaceae } \\
\hline Davilla nitida (Vahl) Kubitzki & - & - & - & Trep & 424 \\
\hline Doliocarpus spraguei Cheeseman & - & - & - & Trep & 421 \\
\hline \multicolumn{6}{|l|}{ Discoreaceae } \\
\hline Discorea melastomotifolia Uline ex Prain & - & - & - & Trep & 420 \\
\hline \multicolumn{6}{|l|}{ Euphorbiaceae } \\
\hline Croton mucronifolius Müll. Arg. & malva-branca & Ago. e Fev. & Fev. & Sub & 4743 \\
\hline Euphorbia hyssopifolia L. & - & Fev. & - & Erva & 4796 \\
\hline Euphorbia sp. ${ }^{1}$ & - & Fev. & Fev. & Erva & 4797 \\
\hline Euphorbia sp. ${ }^{2}$ & - & - & Fev. & Sub & 4798 \\
\hline \multicolumn{6}{|l|}{ Eriocaulaceae } \\
\hline Syngonanthus densiflorus (Körn.) Ruhland & - & Ago. & - & Erva & 5020 \\
\hline Syngonanthus nitens (Bong.) Ruhland & campim-guiné & Nov. & - & Erva & 5111 \\
\hline \multicolumn{6}{|l|}{ Fabaceae } \\
\hline Abrus fruticulosus Weight \& Arn. & - & - & - & Trep & 531 \\
\hline Ancistrotropis firmula (Mart. ex Benth.) A. Delgado & - & Ago. & - & Trep & 5015 \\
\hline Bauhinia sp. & - & Fev. & Fev. & Sub & 4799 \\
\hline Chamaecrista flexuosa (L.) Greene & - & Fev. & Fev. & Sub & 4800 \\
\hline Chamaecrista hispidula (Vahl) H.S.Irwin \& Barneby & - & Ago. & & Erva & 5014 \\
\hline Chamaecrista repens (Vogel) H. S. Irwin \& Barneby & - & Fev. & - & Sub & 4801 \\
\hline Chamaecrista ramosa (Vogel) H. S Trwin \& Barneby & - & Fev. & Fev. & Sub & 4746 \\
\hline Chamaecrista rotundifolia (Pers.) Greene & - & Fev. & Fev. & Erva & 4802 \\
\hline Chamaecrista sp. ${ }^{1}$ & - & Fev. & - & Erva & 4803 \\
\hline Chamaecrista sp. ${ }^{2}$ & - & Fev. & - & Sub & 4804 \\
\hline Crotalaria goreensis Guill \& Perr. & - & Fev. & - & Sub & 4805 \\
\hline Derris sp. & - & - & - & Trep & 529 \\
\hline Dioclea bicolor Benth. & - & Ago. & - & Trep & 4745 \\
\hline Periandra coccinea (Schrader.) Benth. & - & Fev. & - & Trep & 4806 \\
\hline Família/Espécie & Nome Comum & Floração & Frutificação & Hábito & SLUI \\
\hline Periandra heterophylla Benth & - & Fev. & - & Sub & 520 \\
\hline Stylosanthes gracilis Kunth & - & - & - & Sub & 4807 \\
\hline Tephrosia toxicaria (Sw.) Pers. & - & Fev. & Fev. & Sub & 522 \\
\hline Zornia latifolia Sm. & - & - & - & Sub & 4808 \\
\hline \multicolumn{6}{|l|}{ Gentianaceae } \\
\hline Symphyllophyton campos-portoi Gilg-Bem. & - & Ago. e Nov. & - & Erva & 5000 \\
\hline \multicolumn{6}{|l|}{ Iridaceae } \\
\hline Cipura sp. & - & - & - & Erva & 369 \\
\hline Trimezia lutea (Klatt) Foster & - & Fev. & - & Erva & 4809 \\
\hline \multicolumn{6}{|l|}{ Krameriaceae } \\
\hline Krameria tomentosa A.St.-Hil. & - & Ago. & Ago. & Sub & 5016 \\
\hline
\end{tabular}


Tabela 2. Cont

\begin{tabular}{|c|c|c|c|c|c|}
\hline Família/Espécie & Nome Comum & Floração & Frutificação & Hábito & SLUI \\
\hline \multicolumn{6}{|l|}{ Lamiaceae } \\
\hline $\begin{array}{l}\text { Cyanocephalus rugosus (Benth.) Harley \& } \\
\text { J.F.B.Pastore }\end{array}$ & - & Ago. & - & Sub & 4784 \\
\hline Hyptis goyazensis A. St.-Hil. Ex Benth & - & - & - & Sub & 476 \\
\hline Hyptis sp. & - & - & - & Sub & 442 \\
\hline \multicolumn{6}{|l|}{ Lythraceae } \\
\hline Cuphea tenuissima Koehne & - & Fev. & - & Erva & 4810 \\
\hline \multicolumn{6}{|l|}{ Malpighiaceae } \\
\hline Banisteriopsis stellaris (Griseb.) B. Gates & - & Fev. & - & Trep & 4811 \\
\hline Aspicarpa sp. & - & Ago. & - & Sub & 5017 \\
\hline Aspicarpa hirtella Rich. & - & Ago. & - & Trep & 5018 \\
\hline \multicolumn{6}{|l|}{ Malvaceae } \\
\hline Ayenia angustifolia A. ST.-Hil. \& Naudin & malva-roxa & Fev. & - & Sub & 4744 \\
\hline Helicteres sacarolha A. St.-Hil. & - & Fev. & - & Sub & 4812 \\
\hline Melochia hirsute Cav. & - & - & - & Sub & - \\
\hline Sida $\mathrm{sp}^{1}$ & - & Fev. & - & Sub & 4813 \\
\hline Sida $\mathrm{sp}^{2}$ & - & Ago. e Nov. & - & Sub & - \\
\hline Waltheria americana L. & - & - & - & Sub & 570 \\
\hline Malvaceae 1 & - & Ago. & - & Sub & - \\
\hline \multicolumn{6}{|l|}{ Maranthaceae } \\
\hline Monotagma plurispicatum (Körn) K. Schum & - & - & - & Erva & 536 \\
\hline \multicolumn{6}{|l|}{ Mayacaceae } \\
\hline Mayaca fluviatilis Aubl. & - & - & - & Erva & 504 \\
\hline \multicolumn{6}{|l|}{ Melastomataceae } \\
\hline Desmocelis villosa (Aubl.) & - & - & - & Sub & 487 \\
\hline Macairea radula (Bonpl) DC. & - & - & - & Sub & 493 \\
\hline Miconia albicans (Sw.) Steud. & brejo & Nov. & - & Sub & 5110 \\
\hline \multicolumn{6}{|l|}{ Myristicaceae } \\
\hline Virola subsessilis (Benth.) Warb. & - & - & - & Sub & 506 \\
\hline \multicolumn{6}{|l|}{ Ochnaceae } \\
\hline Sauvagesia longifolia Eichler & - & - & - & Sub & - \\
\hline \multicolumn{6}{|l|}{ Passifloraceae } \\
\hline Passiflora glandulosa Cav. & - & - & - & Trep & 549 \\
\hline Piriqueta sp. & - & Ago. & - & Sub & 5131 \\
\hline Turnera brasiliensis Willd. ex Schult. & erva-da-chapada & Nov. & - & Sub & 5132 \\
\hline Turnera caerulea DC. var. caerulea & - & - & - & Sub & 474 \\
\hline Turnera melochioides Cambess. & - & - & - & Sub & 588 \\
\hline \multicolumn{6}{|l|}{ Plantaginaceae } \\
\hline Conobea aquatica Aubl. & - & - & - & Erva & - \\
\hline \multicolumn{6}{|l|}{ Poaceae } \\
\hline Astrostylidium sp & - & - & - & Erva & 461 \\
\hline Axonopus pubivaginatus var limae G. A. Black & - & - & - & Erva & 452 \\
\hline Paspalum gardnerianum Nees & - & - & - & Erva & - \\
\hline Paspalum serpentinum Hochst. exSteud & - & - & - & Erva & - \\
\hline Trachypogon spicatus (L. F.) Kuntze & - & - & - & Erva & - \\
\hline \multicolumn{6}{|l|}{ Polygalaceae } \\
\hline Asemeia violacea (Aubl.) J.F.B.Pastore \& J.R.Abbott & - & Fev. & - & Sub & 4814 \\
\hline Securidaca longifolia Poepp. \& Endl. & - & Ago. & - & Trep & 5019 \\
\hline
\end{tabular}


Tabela 2. Cont.

\begin{tabular}{|c|c|c|c|c|c|}
\hline Família/Espécie & Nome Comum & Floração & Frutificação & Hábito & SLUI \\
\hline Moutabea guianensis Aubl. & - & - & - & Trep & 550 \\
\hline \multicolumn{6}{|l|}{ Portulacaceae } \\
\hline Portulaca elatior Mart. Ex Rohrb. & - & Fev. & - & Erva & 4815 \\
\hline Portulaca mucronata Link & - & Fev. & - & Erva & 4816 \\
\hline \multicolumn{6}{|l|}{ Rubiaceae } \\
\hline Borreria tenera DC. & - & - & - & Erva & 557 \\
\hline Borreria verticillata (L.) G.Mey. & - & Fev. & - & Erva & 4817 \\
\hline $\begin{array}{l}\text { Declieuxia fruticosa (Willd. ex Roem. \& Schult.) } \\
\text { Kuntze }\end{array}$ & - & Fev. & - & Sub & 4818 \\
\hline Hexasepalum teres (Walter) J.H. Kirkbr & - & Fev. & - & Erva & 4819 \\
\hline Mitracarpus hirtus (L.) DC. & - & Fev. & - & Erva & 4820 \\
\hline \multicolumn{6}{|l|}{ Sapindaceae } \\
\hline Serjania grandifolia Sagote $\mathrm{x}$ Radlk & - & - & - & Trep & 581 \\
\hline \multicolumn{6}{|l|}{ Smilacaceae } \\
\hline Smilax salicifolia Griseb & - & - & - & Trep & 576 \\
\hline \multicolumn{6}{|l|}{ Verbenaceae } \\
\hline Lippia gracilis Schauer & - & - & - & Erva & - \\
\hline \multicolumn{6}{|l|}{ Xyridaceae } \\
\hline Xyris macrocephala Vahl & - & - & - & Erva & 599 \\
\hline
\end{tabular}

$\mathrm{N}^{\circ}$ de registro= é estabelecido pelo Herbário Rosa Mochel (SLUI) da Universidade Estadual do Maranhão, onde as espécies foram armazenadas.

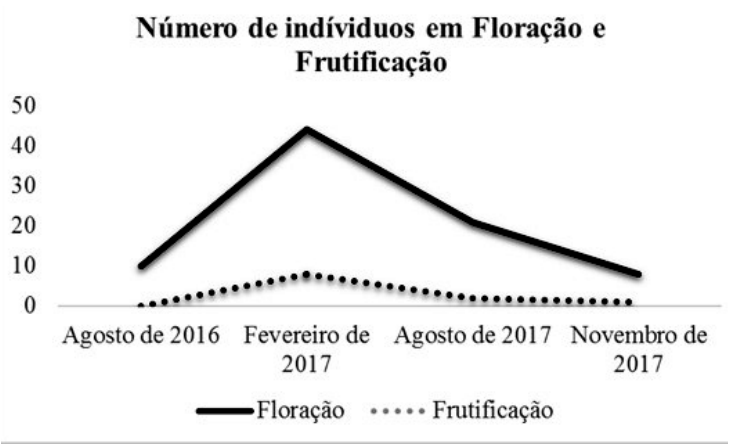

Figura 3. Número de espécies com presença de flores e frutos de acordo com o período de coleta.

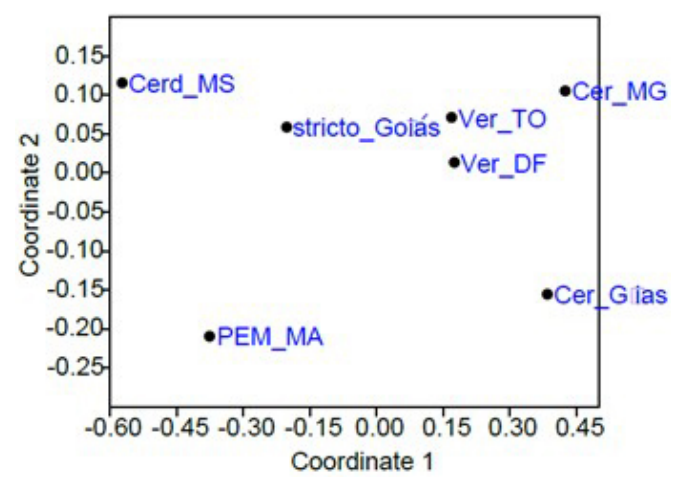

Figura 4. Análise de similaridade (Índice de Jaccard) entre áreas do cerrado brasileiro. Parque Estadual do Mirador/Maranhão (PEM_MA); valor de stress: 0.06266 . Cerd_MS = Cerradão do Mato Grosso do Sul; stricto_Goiás = cerrado sensu stricto de Góias; _DF $=$ Vereda do Distrito Federal; Cer_Goiás = cerrado de Goiás.
Segundo a Flora do Brasil 2020 e ausência de registro em trabalhos publicados sobre flora maranhense ou tratamentos taxonômicos, 11 espécies catalogadas neste trabalho possuem os primeiros registros para o estado do Maranhão, sendo as seguintes: Aspilia leucoglossa Malme, Ichthyothere latifolia, Bonamia agrostopolis Vell., Evolvulus frankenioides Moric., Evolvulus pusillus, Crotalaria goreensis Guill \& Perr., Helicteres sacarolha A. St.-Hil, Mitracarpus hirtus (L.) DC, Symphyllophytton camposportoi, Syngonanthus nitens (Bong.) Ruhland, Securidaca longifolia Eichler (Figs. 5, 7 e 8) que corresponde a 19,04\% das plantas coletadas no PEM. Os primeiros registros para o domínio cerrado foram: Habranthus sylvaticus Herb., Evolvulus anagalloides, Crotalaria goreensis e Securidaca longifolia.

Das 52 espécies identificadas a nível específico, duas são endêmicas do domínio Cerrado (3,85\%) e 96,15\% (50 espécies) são compartilhadas com outros domínios. Se considerarmos as espécies compartilhadas exclusivamente com cada domínio fitogeográfico brasileiro, em um ranking temos: Caatinga com 10\% (cinco espécies), Amazônia 6\% (três espécies), Mata Atlântica 6\% (três espécies) e 78\% das espécies estão presentes em mais de dois domínios fitogeográficos. Teixeira (2015) encontraram essa mesma distribuição de compartilhamento entre os domínios em estudos no Cerrado stricto sensu. A presença de espécies da flora da Caatinga em áreas de Cerrado também foi verificada por Françoso (2014) e Teixeira (2015). 

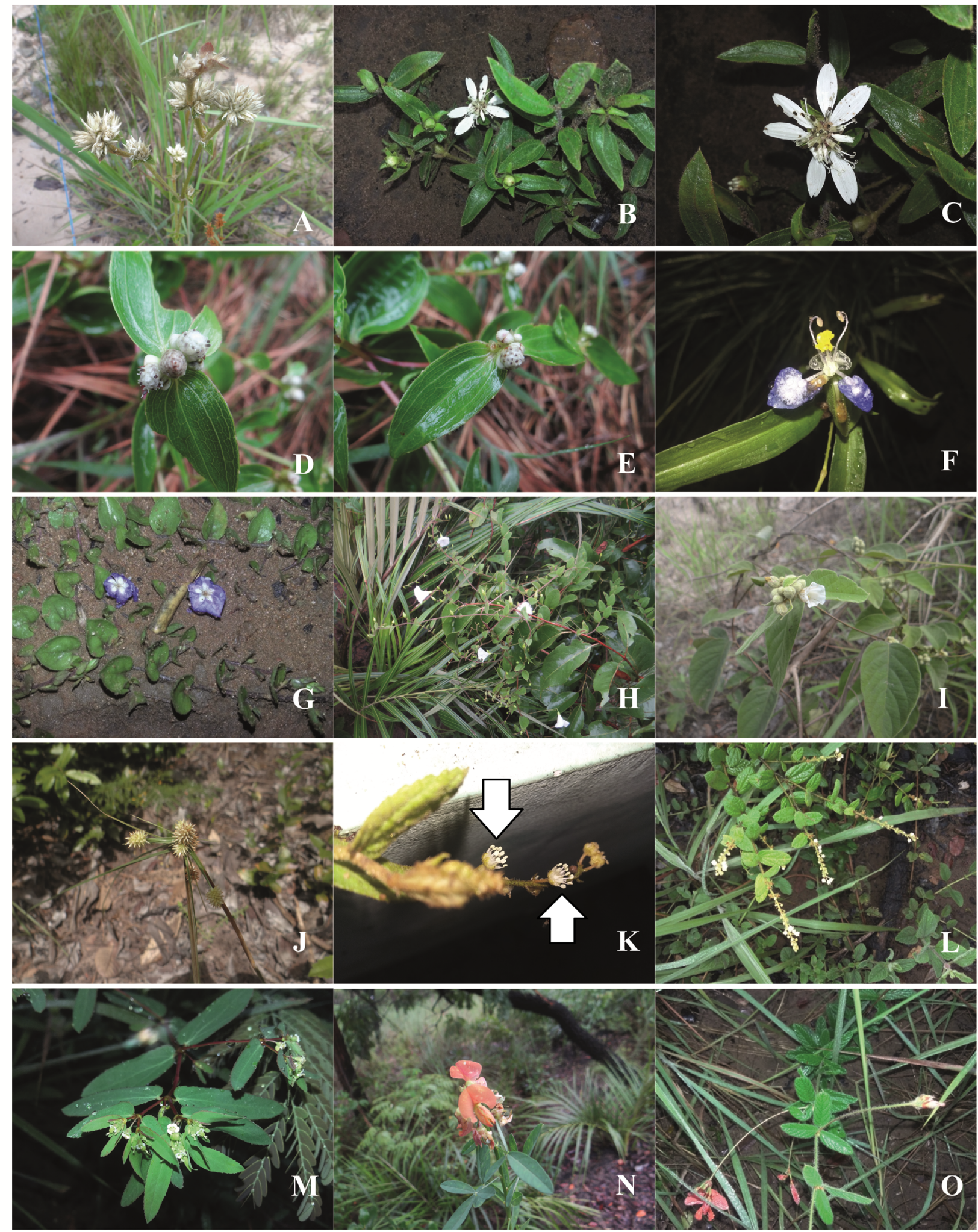

Figura 5. Imagens das espécies coletadas no Parque Estadual do Mirador. A. Gomphrena agrestis Mart.; B-C. Aspilia leucoglossa Malme; D-E. Ichthyothere latifolia Baker; F. Commelina erecta L.; G. Evolvulus anagalloides Meisn.; H. Evolvulus pusillus Choisy; I. Bonamia agrostopolis (Vell.) Hallier; J. Cyperus sp.; K-L. Croton mucronifolius Müll. Arg.; M. Euphorbia hyssopifolia L.; N. Crotalaria goreensis Guill. \& Perr.; O. Periandra coccinea (Schrader.) Benth. 

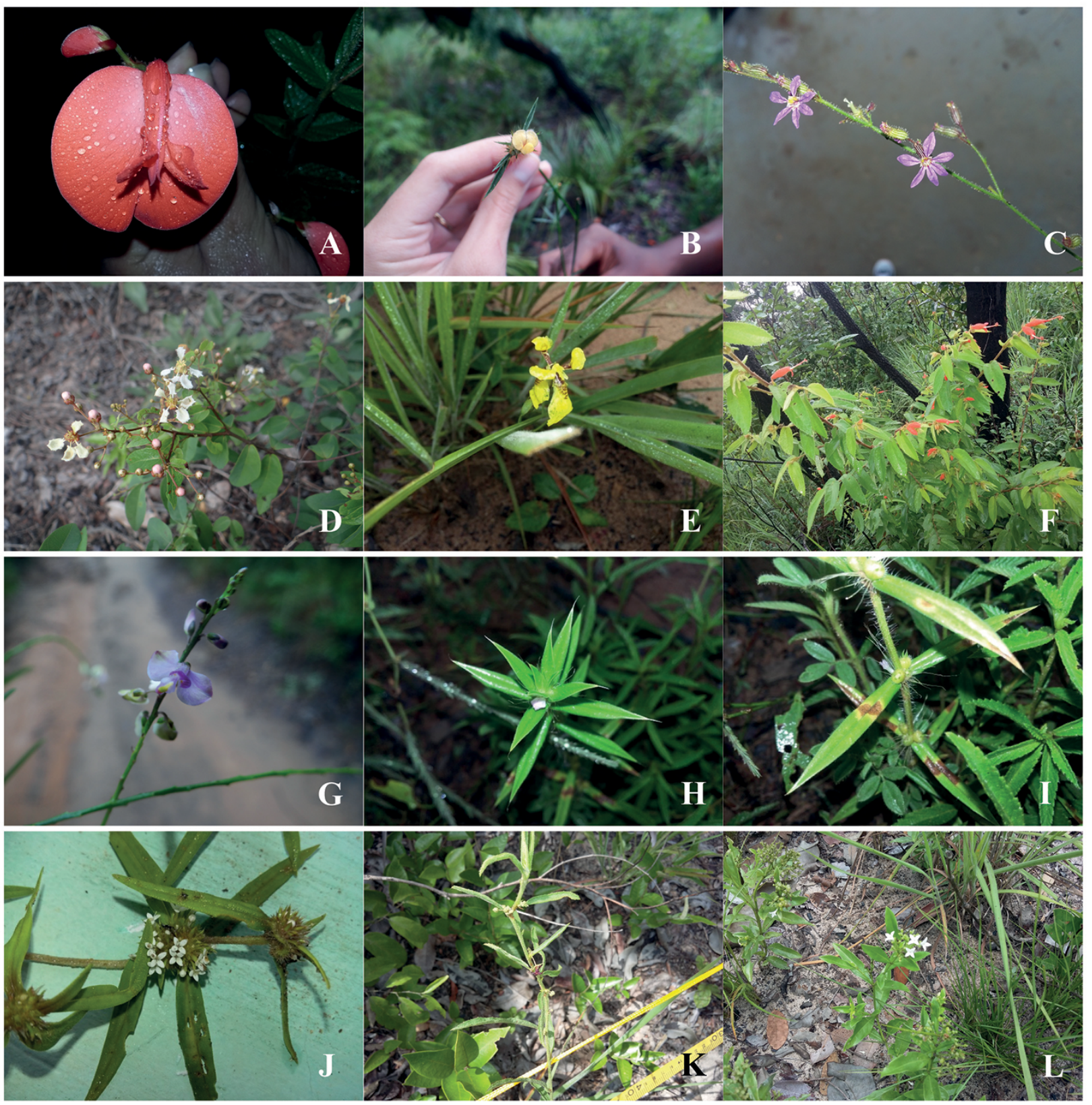

Figura 6. Imagens das espécies coletadas no Parque Estadual do Mirador (PEM). A. Periandra coccinea (Schrader.) Benth.; B. Stylosanthus gracilis Kunth.; C. Cuphea tenuissima Koehne; D. Banisteriopsis stellaris (Griseb.) B. Gates; E. Trimezia lutea (Klatt) Foster; F. Helicteres sacarolha A. St.-Hil.; G. Asemeia violacea (Aubl.) J.F.B. Pastore \& J.R. Abbott; H-I. Diodella teres (Walter) Small; J. Mitracarpus hirtus (L.) DC.; K. Ayenia angustifolia A. ST.-Hil. \& Naudin; L. Declieuxia fruticosa (Willd. ex Roem. \& Schult.) Kuntze.

A composição florística para o período seco no Parque Estadual do Mirador apresentou um número reduzido de espécies em floração/frutificação (Fig. 3). Meirelles et al. (1999) em trabalho no Cerrado do Munícipio de Balsas do Maranhão destacaram que na estação seca, o número de espécies do estrato herbáceo-subarbustivo é reduzido devido as plantas terem perdido sua parte aérea. A vegetação herbácea detém característica de vida subterrânea, voltando a emergir ramos quando as condições climáticas forem favoráveis (período chuvoso). Espécies catalogadas neste trabalho, também foram identificadas por Rodrigues \& Conceição (2014) em levantamentos florísticos no Parque Estadual do Mirador, em relação ao componente herbáceo.
Referente ao período chuvoso foi inventariado um maior número de espécies, devido às condições ambientais ideais para o desenvolvimento da vegetação herbáceosubarbustiva. Munhoz \& Felfili (2006) destacaram, em estudos realizados em fitofisionomia em campo sujo no Distrito Federal, que algumas espécies na estação seca perdem sua parte aérea e somente serão inventariadas ao longo do período chuvoso, e enfatizam que os trabalhos envolvendo o componente herbáceo-subarbustivo devem ser realizados preferencialmente na estação chuvosa para abranger um maior número de espécies no inventário florístico, e neste trabalho foi possível realizar apenas uma coleta no período chuvoso em decorrência deste fator 


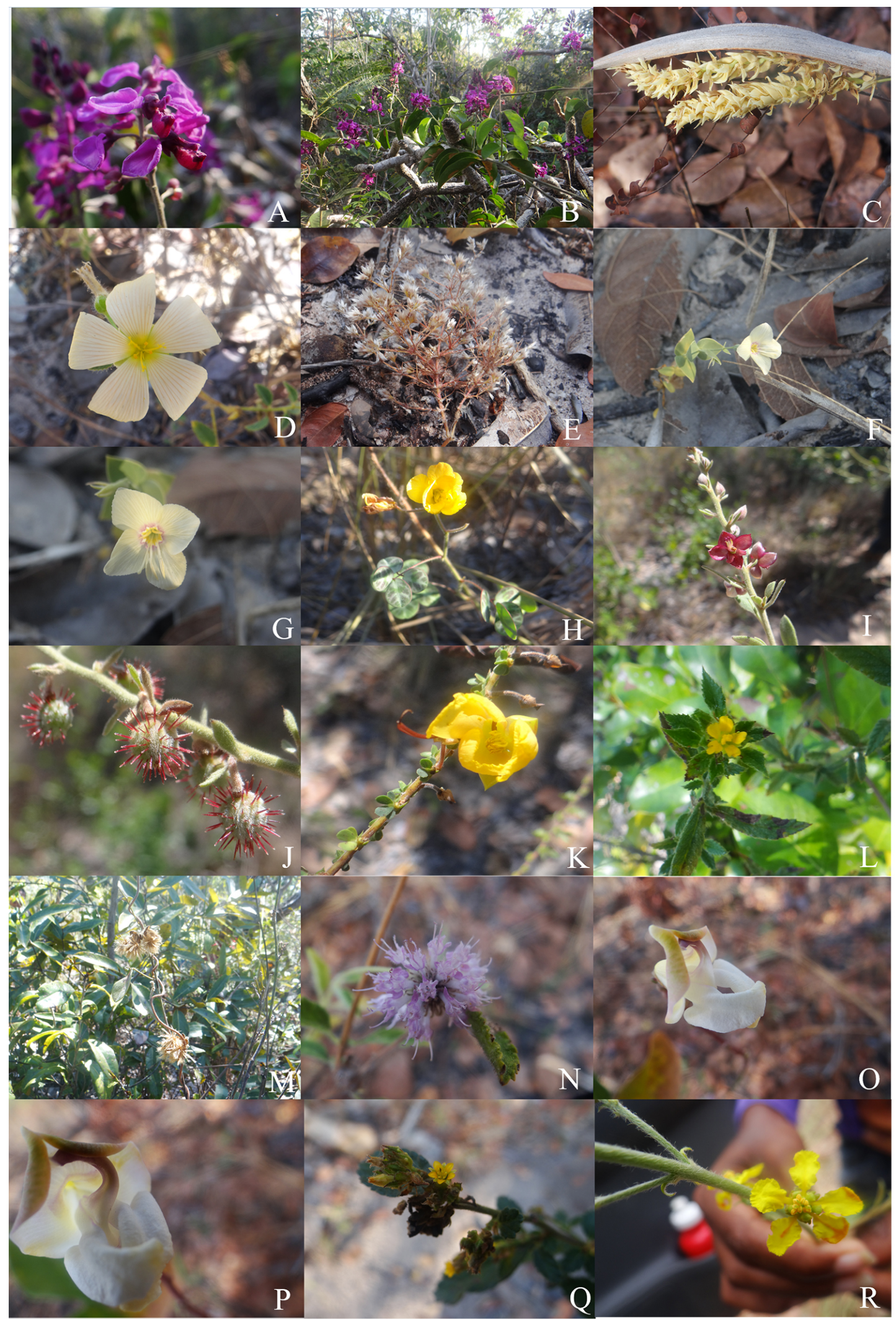

Figura 7. Imagens das espécies catalogadas no Parque Estadual do Mirador (PEM). A-B. Securidaca longifolia Poepp. \& Endl.; C. Syagrus comosa (Mart.) Mart.; D. Piriqueta sp; E. Polycarpea corymbosa (L.) Lam.; F-G. Symphyllophyton sp; H. Chamaecrista hispidula (Vahl) H.S.Irwin \& Barneby; I. Krameria tomentosa A.St.-Hil.; J. Krameria tomentosa A.St.-Hil.; K. Chamaecrista ramosa (Vogel) H. S Trwin \& Barneby; L. Sida sp2 M. Vernonia sp; N. Cyanocephalus rugosus (Benth.) Harley \& J.F.B.Pastore; O-P. Ancistrotropis firmula (Mart. ex Benth.) A. Delgado; Q. Malvaceae; R: Aspicarpa sp. 

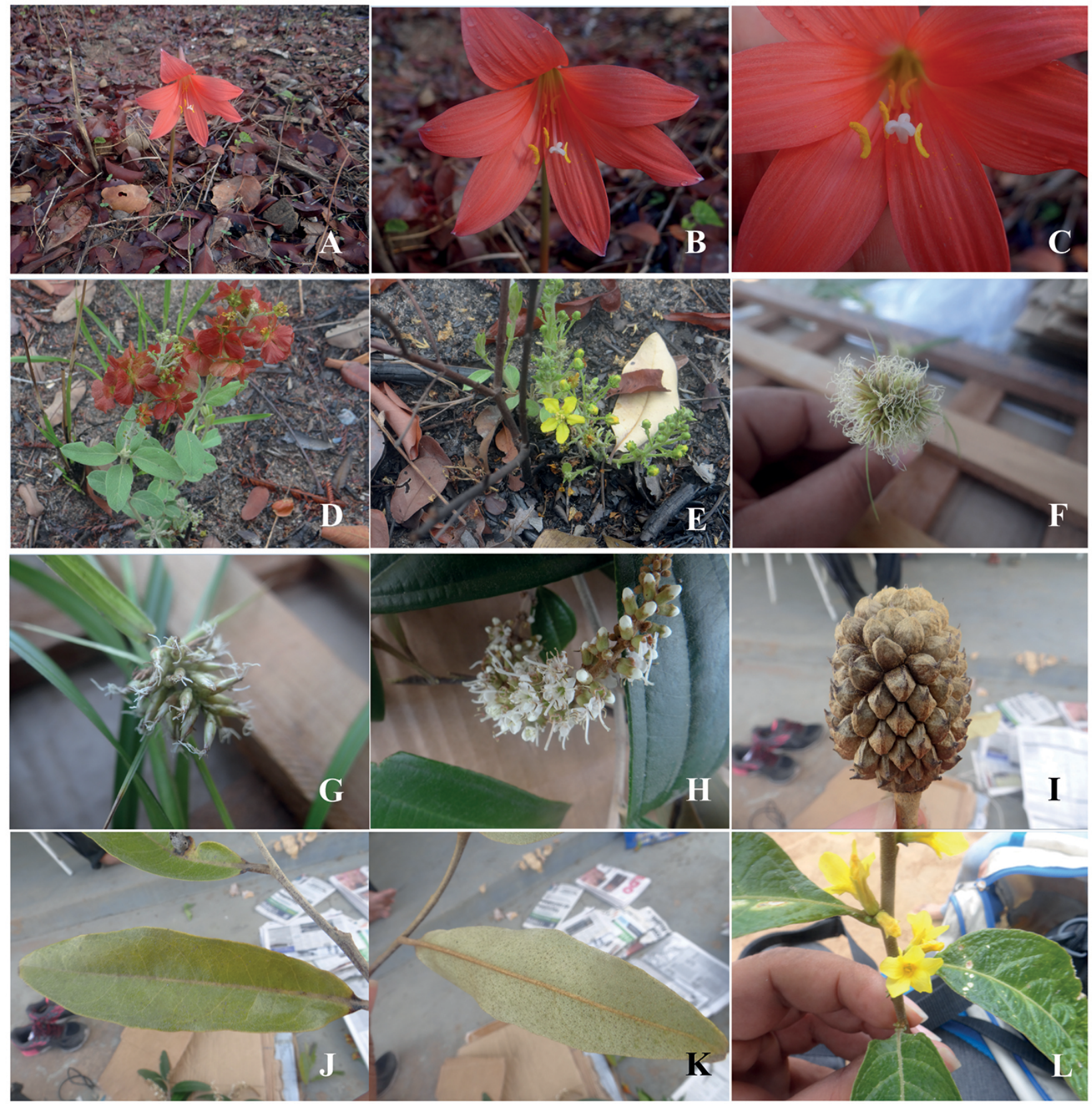

Figura 8. Imagens das espécies catalogadas no Parque Estadual do Mirador (PEM), A-C. Habranthus sylvaticus Herb.; D-E. Aspicarpa sp; F. Rhynchospora cephalotes (L.) Vahl; G. Rhynchospora sp; H. ,Miconia albicans (Sw.) Steud.; I-K. Anonnaceae; L. Turnera brasiliensis Willd. ex Schult.

muitas espécies não foram inventariadas, sejam estágios de floração ou frutificação. $\mathrm{Na}$ área de coleta foi observado uma dominância da espécie Trachypogon spicatus (L. F.) Kuntze na área de Cerrado stricto sensu do Parque Estadual do Mirador que também foi relatado por Silva et al. (2020) para a mesma área e por Farias \& Mendes (2017) no Piauí no Parque Nacional das Setes Cidades em área de Cerrado stricto sensu e essa espécie tem característica de apresentar uma maior tolerância ao estresse hídrico e este fator pode justificar sua dominância na área em relação as outras espécies (Silva \& Klink 2011, Farias \& Mendes 2017).

A similaridade feita entre áreas do Cerrado mostrou diferença na composição de espécies coletadas no Parque
Estadual do Mirador comparado a outros trabalhos publicados com estrato herbáceo-subarbustivo. É possível evidenciar que a flora constituinte do Cerrado stricto sensu do PEM, do cerradão em Mato Grosso do Sul e do Cerrado em Goiás apresentaram baixa similaridade com as outras áreas que ficaram mais próximas, estando representado no espaço multidimensional não métrico (Fig. 4).

A separação florística entre áreas é influenciada pelos tipos de fisionomias, embora a flora do Cerrado pareça homogênea, os estudos revelam uma heterogeneidade nas amostras (Silva et al. 2017). Através de comparações de similaridade é possível ver a heterogeneidade do Cerrado entre as fitofisionomias. Essa distinção também 
é associada a fatores climáticos e ambientais que atuam diretamente sobre a vegetação e no seu estabelecimento no ambiente. Rizzini (1976) destaca que essa distinção florística pode estar atrelada a ideia de "zona marginal" e de "individualidade fitogeográfica do Cerrado maranhensepiauiense". Castro (1994) e Castro et al. (2007) acentuam que os Cerrados do Maranhão e Piauí estão localizados na região marginal de distribuição do Cerrado no território brasileiro, em vez de "individualidade florística" empregado por Rizzini (1976), estes autores apresentam a ideia da existência de um supercentro de biodiversidade do Cerrado no nordeste, e que a separação do Cerrado em três supercentros de biodiversidade estaria relacionada às barreiras climáticas entre outros fatores ambientais, que podem influenciar na composição de espécies do Cerrado e nessa distinção florística entre as áreas, como o Polígono das Secas e o Polígono das Geadas, ou às cotas altimétricas de $400 / 500 \mathrm{~m}$ e de $900 / 1.000 \mathrm{~m}$. Em um estudo preliminar feito por Castro et al. (2007) através de uma base de dados denominada FLORACENE obtida a partir de análises fitossociológicas rápidas para o componente arbóreo do Cerrado do Nordeste, confirmaram a existência de uma flora areal distinta, mas evidenciado que a flora dessas regiões ainda são subamostradas e que está hipótese precisam de mais dados para ser testada. Este trabalho engloba a primeira listagem da vegetação herbáceo-subarbustiva para o Cerrado Maranhense e o Parque Estadual do Mirador, havendo anteriormente somente dados florísticos para a vegetação arbórea.

\section{AGRADECIMENTOS}

A Universidade Estadual do Maranhão Campus Paulo VI, Rede ComCerrado e a FAPEMA pelo financiamento do projeto e ao $\mathrm{CNPq}$ pela bolsa de Iniciação Científica concedida ao primeiro autor. À equipe do Herbário Rosa Mochel, pelos auxílios em campo e no herbário.

\section{REFERÊNCIAS}

Alcântara, E.H. 2004. Caracterização da bacia hidrográfica do rio Itapecuru, Maranhão Brasil. Caminhos de Geografia 7:97-113.

Amaral, A. G., Munhoz, C.B.R., Eugênio, C.U.O. \& Felfili, J.M. 2013. Vascular flora in dry-shrub and wet grassland Cerrado seven years after a fire, Federal District, Brasil. Check List 9:487-503.

Amaral, A. G., Munhoz, C.B.R., Walter, B. M.T., Aguirre-Gutiérrez, J. \& Raes, N. 2017. Richness pattern and phytogeography of the Cerrado herb-shrub flora and implications for conservation. Journal of Vegetation Science 28:848-858.

Andrade, E. B.; Weber, L. N. \& Leite, J. R. S. A. 2017. Anurans of the Parque Estadual do Mirador, a remnant of Cerrado in the state of Maranhão, Northeastern Brazil. Biota Neotropica 17:1-12.

Andrella, G. C. \& José Neto, M. 2017. Levantamento florístico dos arredores do Parque das Capivaras, Três Lagoas-MS. Revista Saúde e Meio Ambiente - Resma, Três Lagoas 5:70-77.

Batalha, M.A. \& Mantovani, W. 2001. Floristic composition of the cerrado in the pé-de-gigante reserve (Santa Rita do Passa Quatro, Southeastern Brazil). Acta botanica brasílica 15:289-304.

Bijos, N. R., Eugênio, C.U.O., Melo, T.R.B., Souza, G.F. \& Munhoz, C.B.R. 2017a. Plant species composition, richness, and diversity in the palm swamps (veredas) of Central Brazil. Flora, 237:94-99.
Bijos, N. R. 2017b. Fatores modeladores da composição e da diversidade de espécies herbáceo-arbustivas em veredas no Brasil. Dissertação 92 f., Curso de Botânica, Instituto de Ciências Biológicas, Universidade de Brasília, Brasília.

Byng, J. W., Chase, M. W, Christenhusz, M. J. M., Fay, M. F., Judd, W. S., MabberleY, D. J., Sennikov, A. N., Soltis, D. E., Soltis, P. S., Stevens, P. F. 2016. An update of the Angiosperm Phylogeny Group classification for the orders and families of flowering plants: APG IV. Botanical Journal of The Linnean Society 181:1-20.

Cardoso, D. B. O. S. e Queiroz, L. P. 2010. Caatinga no contexto de uma metacomunidade: evidências da biogeografia, padrões filogenéticos e abundância de espécies em leguminosas. In Biogeografia da América do Sul: padrões e processos (C. J.B. Carvalho \& E. Almeida, eds) Roca, São Paulo, p. 241-260.

Carvalho, F. A., Rodrigues, V.H.P., Kilca, R.V., Siqueira, A. S., Araújo, G.M. 2008. Composição florística, riqueza e diversidade de um cerrado sensu stricto no sudeste do estado de Goiás. Biosciense Journal 24:64-72.

Castro, A.A.J.F. 1994. Comparação florística de espécies do cerrado. Silvicultura 15:16-18.

Castro, A.A.J.F., Castro, N.M.C.F., Costa, J.M., Farias, R.R.S., Mendes, M.R.A., Albino, R.S., Barros, J.S. Oliveira, M.E.A. 2007. Cerrados Marginais do Nordeste e Ecótonos Associados. Revista Brasileira de Biociências 5:273-275.

Chacon, R. G., Martins, R. C. \& Amaral, A. G. 2014. Checklist da flora herbáceo-arbustiva da Reserva Biológica do Guará, Distrito Federal: levantamento da flora como parte integrante de estudos para subsidiar o manejo e gestão de áreas protegidas. Heringeriana 8:81-122.

Conceição, G.M. \& Castro, A.A.J.F. 2009. Fitossociologia de uma área de Cerrado Marginal, Parque Estadual do Mirador, Mirador, Maranhão. Scientia Plena 5:1-16.

Embrapa-Empresa Brasileira de Pesquisa Agropecuária. 2012. Comunicado Técnico 144: Leguminosas florestais da Mata Atlântica brasileira fixadoras de nitrogênio atmosférico. 1 ed. Seropédica, Rio de Janeiro, p.13.

Fernandes, J. M. \& Garcia, F. C. P. 2008. Leguminosae em dois fragmentos de floresta estacional semidecidual em Araponga, Minas Gerais, Brasil: arbustos, subarbustos e trepadeiras. Rodriguésia 59:525-546.

Françoso, R. D. 2014. Padrões biogeográficos e composição das comunidades arbóreas do cerrado brasileiro. Tese $150 \mathrm{f}$., Universidade de Brasília, Brasília.

Maranhão. Lei $\mathrm{n}^{\circ} 8.958$ de 08 de maio de 2009. Altera o Decreto $\mathrm{n}^{\circ}$ $7.641 / 80$ de junho de 1980, que cria o Parque Estadual de Mirador e dá outras providências. São Luís: D.O.E, de 08.05.2009, Ano CIII, n. 087.

Mccune, B. \& Grace J. B. 2002. Analysis of Ecological Communities. Gleneden Beach (USA): MjM Software Design, ISBN 0972129006.

Meirelles, M. L., Oliveira, R.C., Ribeiro, J.F., Vivaldi, L.J., Rodrigues, L.A., Silva, G.P. 1999. Levantamento do estrato herbáceo do cerrado utilizando o método de intersecção na linha. Empresa Brasileira de Pesquisa Agropecuária 1:1-2.

Mello, T. R. B. 2012. Comunidades herbáceo-arbustivas e suas relações com solo e altitude, em áreas secas e úmidas, no Parque Nacional das Sempre Vivas, MG. Dissertação 62 f., Universidade de Brasília, Brasília.

Mendonça, R.C., Felfili, J.M., Walter, B.M.T., Silva Júnior, M.C., Rezende, A.V., Filgueiras, T.S., Nogueira, P.E., Fagg, C.W. 2008. Flora Vascular do Cerrado. In Cerrado: ambiente e flora. (S.M. Sano, S.P. Almeida, eds). Empresa Brasileira de Pesquisa Agropecuária-CPAC, Brasília, p. $289-556$

Mendonça, R.C., Felfili, J.M, Fagg, C.W., Silva, M.A., Filgueiras, T.S., Walter, B.M.T. 2001. Análise florística da Chapada do Espigão Mestre de São Francisco. In Biogeografia do bioma cerrado: estudo fitofisionômico na Chapada do Espigão Mestre do São Francisco (J.M. Felfili, M.C. Silva Júnior, eds) Editora Universidade de Brasília, Brasília, p. 74-79.

Moro, M. F. \& Martins, F. R. 2011. Métodos de levantamento do componente arbóreo-arbustivo. In Fitossociologia no Brasil: métodos e estudos de caso (J. M. Felfili, P. V. Eisenlohr, M. M. R. F. M., et al. eds). Universidade Federal de Viçosa, Viçosa. 177 p. 
Munhoz, C. B. R. \& Felfili, J.M. 2005. Fenologia do estrato herbáceosubarbustivo de uma comunidade de campo sujo na Fazenda Água Limpa no Distrito Federal, Brasil. Acta Botanica brasilica 19:981-990.

Munhoz, C. B. R \& Felfili, J. M. 2006. Fitossociologia do estrato herbáceosubarbustivo de uma área de campo sujo no Distrito Federal, Brasil. Acta Botanica brasilica 20:671-685.

Munhoz, C. B. R. \& Felfili, J. M. 2007. Florística do estrato herbáceosubarbustivo de um campo limpo úmido em Brasília, Brasil. Biota Neotropica 7:206-214

Muniz, F. H. 2006. A vegetação da região de transição entre a Amazônia e o Nordeste: Diversidade e Estrutura. Universidade Estadual do Maranhão, São Luís, 354 p.

REFLORA: Flora do Brasil 2020. Disponível em: http://reflora.jbrj. gov.br/reflora/listaBrasil/ConsultaPublicaUC/ConsultaPublicaUC. do\#CondicaoTaxonCP. Acessado em: 22.11.2016.

Rezende, J. M. 2007. Florística, fitossociologia e a influência do gradiente de umidade do solo em Campos Limpos Úmidos no Parque Estadual do Japalão-Tocantins. Dissertação 74 f., Universidade de Brasília, Brasília.

Rios, M. N. da S. 2016. Dinâmica de comunidades vegetais em cerrado típico com histórico de fogo no Distrito Federal. Tese $175 \mathrm{f}$., Universidade de Brasília, Brasília.

Rizzini, C.T. de. 1976. Contribuição ao conhecimento das floras nordestinas. Rodriguesia 28:137-193.

Rodrigues, M. S. \& Conceição, G. M. 2014. Diversidade Florística das diferentes fitofisionomias de Cerrado do Parque Estadual do Mirador
Maranhão, Brasil. Brazilian Geographical Journal: Geo sciences and Humanities research medium 5:139-156.

Secretaria de Meio Ambiente e Recursos Naturais. Unidades de Conservação. 2017. Disponível em: http://www.sema.ma.gov.br/ conteudo?/430/Unidades_de_Conservação. Acesso em: 08 de maio de 2018.

Silva, D. P. 2016. Composição, estrutura e diversidade da vegetação herbáceo-arbustiva em veredas no Jalapão, Tocantins. Dissertação 76 f., Universidade de Brasília, Brasília.

Silva, D. P., Amaral A. G., Bijos, N.R., Munhoz, C.B.R. 2017. Is the herb-shrub composition of veredas (Brazilian palm swamps) distinguishable? Acta Botanica Brasilica 32:47-54.

Tannus, J.L.S; Assis, M. A. 2004. Composição de espécies vasculares de campo sujo e campo úmido em área de cerrado, Itirapina - SP, Brasil. Revista Brasileira de Botânica 27:489-506.

Teixeira, A. M. C. 2015. Florística e estrutura da vegetação em Cerrado sentido restrito no Parque Estadual de Terra Ronca, Goiás: método RAPELD. Dissertação 102 f., Universidade de Brasília, Brasília.

Teixeira, A.M.C., Pinto, J.R. Amaral, A. G., Munhoz, C.B.R. 2016 Angiosperm species of "Cerrado" sensu stricto in Terra Ronca State Park, Brazil: floristics, phytogeography and conservation. Brazilian Journal Of Botany 40:225-234.

Tropicos-Namesearch. Disponível em: http://www.tropicos.org/ NameSearch.aspx. Acessado em: 8.01.2017.

Whittaker, R. H. 1975.Communities and ecossystems. MacMillan 2:385. 